\title{
N $94-22814$
}

\section{DS-SSMA capacity for a mobile satellite system}

\author{
Francesco Bartucca Ezio Biglieri* \\ Dipartimento di Elettronica, Politecnico, Corso Duca degli Abruzzi 24, I-10129 Torino (Italy). Tel: +39 115644030, \\ Fax: +39115644099, e-mail: biglieri@polito.it
}

\begin{abstract}
We consider a cellular satellite system conceived to enhance the capabilities of the pan-European terrestrial system (GSM). This adopts EHF band and highly-inclined orbits. We present a preliminary assessment of system capacity based on asynchronous direct-sequence spreadspectrum multiple access (DS-SSMA). Performance is measured in terms of error probability achieved by $K$ users simultaneously accessing the system with a given signalto-noise ratio.
\end{abstract}

\section{INTRODUCTION}

A cellular satellite system has recently been proposed $[3,4,12]$ which is conceived to enhance the capabilities of the pan-European terrestrial cellular system (GSM). Intended as an efficient complement to GSM, this new system adopts EHF band (specifically, 40/ $50 \mathrm{GHz}$ ), and highly-inclined orbits. Its main goals are: $[3,12]$ (i) To provide higher source data rates (64 $\mathrm{kbit} / \mathrm{s}$, and possibly $n \times 64 \mathrm{kbit} / \mathrm{s}$ ) in order to support ISDN-compatible teleservices. (ii) To expedite the diffusion of mobile services in Europe and expand their coverage to Eastern Europe, part of North Africa, and the Near East. (iii) To integrate maritime and aeronautical users into the terrestrial network. (iv) To offer private users a solution for implementation of mobileradio closed networks with coverage radii up to a few hundred kilometers. (v) To allow implementation of networks of small, portable terminals in the perspective of a forthcoming worldwide personal communication system.

In this paper we present a preliminary assessment of system capacity based on asynchronous direct-sequence spread-spectrum multiple access (DSSSMA). System performance is measured in terms of error probability achieved by $K$ users simultaneously accessing the system with a given signal-to-noise ratio.

\section{SYSTEM MODEL}

In the model of an asynchronous DS-SSMA sys-

*This work was performed for ASI (Italian Space Agency). tem [13]; $K$ users transmit digital data over a common satellite channel with center frequency $f_{c}$. The signal transmitted by the $k$ th user is

$$
s_{k}(t)=\sqrt{2 P} b_{k}(t) a_{k}(t) \cos \left(2 \pi f_{c} t+\theta_{k}\right)
$$

where $P$ denotes the common transmitted power, $b_{k}(t)$ is the data-bearing signal, $a_{k}(t)$ is a periodic waveform with period $T$ and formed by $N$ rectangular chips, each with duration $T_{c}=T / N$ and amplitude \pm 1 , and $\theta_{k}$ is the carrier phase. The signal $b_{k}(t)$ is a rectangular waveform taking on the value $b_{k, l}$ in the time interval $[l T,(l+1) T]$, where $b_{k, l}$ the $l$ th source bit from user $k$, taking on values \pm 1 with equal probabilities and independently of the other $b_{i, j}$.

The code sequence assigned to the $k$ th user takes on value $a_{k}^{(j)}$ in the interval $\left[j T_{c},(j+1) T_{c}\right]$ for a code word $a_{k}^{(0)}, a_{k}^{(1)}, \ldots, a_{k}^{(N-1)}$ taking values \pm 1 . The signal bandwidth is proportional to $T_{c}^{-1}=N / T$, and hence $N$ is the bandwidth-expansion factor.

The signal received by the $k$ th user is

$$
\sqrt{2 P} \sum_{k=1}^{K} a_{k}\left(t-\tau_{k}\right) b_{k}\left(t-\tau_{k}\right) \cos \left(2 \pi f_{c} t+\phi_{k}\right)+n_{k}(t)
$$

where $n_{k}(t)$ is a white Gaussian noise process independent of the other random variables (RV) and whose two-sided power spectral density is $N_{0} / 2$. The delay $\tau_{k}$ and the phase $\phi_{k}=\theta_{k}-2 \pi f_{c} \tau_{k}$ model asynchronous transmission. We assume that $\tau_{k}$ is uniformly distributed in $[0, T], \phi_{k}$ is uniformly distributed in $[0,2 \pi]$, and $\tau_{k}, \phi_{k}$ are independent of each other and of the other RVs in this model.

The $k$ th-user receiver computes the correlation between the received signal and $a_{k}(t) \cos \left(2 \pi f_{c} t\right)$ in the time interval $[0, T]$, and makes a decision on the value of $b_{k, 0}$ based on the sign of this correlation. Consider receiver \#1, and with no loss of generality assume $\tau_{1}=\phi_{1}=0$. With $f_{c} \gg 1 / T$, the correlator output is

$$
Z_{1}=\sqrt{\frac{P}{2}} T b_{1,0}+\sqrt{\frac{P}{2}} u+n,
$$

where the first term in the right-hand side is the useful signal, the second term is the interference from 
the other users, and $n$ is white Gaussian noise. The multiple-access interference RV $u$ is defined as

$$
\begin{aligned}
u & =\sum_{k=2}^{K}\left[b_{k,-1} R_{k, 1}\left(\tau_{k}\right)+b_{k, 0} \hat{R}_{k, 1}\left(\tau_{k}\right)\right] \cos \phi_{k}= \\
& =\sum_{k=2}^{K} w_{k} \cos \phi_{k}
\end{aligned}
$$

where $R_{k, 1}$ and $\hat{R}_{k, 1}$ are partial crosscorrelations

$$
\begin{aligned}
& R_{k, 1}(\tau)=\int_{0}^{\tau} a_{k}(t-\tau) a_{1}(t) \mathrm{d} t \\
& \hat{R}_{k, 1}(\tau)=\int_{\tau}^{T} a_{l}(t-\tau) a_{1}(t) \mathrm{d} t
\end{aligned}
$$

with $0 \leq \tau \leq T$.

The average error probability for user \#1 can be evaluated by using the moments of this RV $u$.

Assume $b_{1,0}=1$. Then the average error probability $P(E)$ is equal to $P\left(Z_{1}<0\right)$, and we have

$$
P(E)=\int Q\left[\sqrt{\frac{2 P T}{N_{0}}}\left(1+\frac{u}{T}\right)\right] f_{u}(u) \mathrm{d}
$$

where $P T / N_{0} \triangleq E_{b} / N_{0}$ is the signal-to-Gaussian noise power ratio, and $E_{b} \triangleq P T$ is the energy per bit. The function $Q(x)$ is defined in the usual way:

$$
Q(x)=\frac{1}{\sqrt{2 \pi}} \int_{x}^{\infty} \exp \left(-t^{2} / 2\right) \mathrm{d} t .
$$

Once the moments of the RV $Z_{1}$ have been evaluated, several techniques are available to compute the error probability (5). Refs. [1, 2, 14] summarize some of them, based on series expansions or on Gauss quadrature rules. In the following we shall use a technique advocated in [13] and based on Gram-Charlier series.

\section{Gram-Charlier expansion}

Gram-Charlier series expand a probability density function (pdf) in terms of derivatives of a known pdf [5].

Following [13], define a RV $x$ associated with multiple-access interference and Gaussian noise:

$$
x=x_{0}\left(\frac{T}{u}+\sqrt{\frac{2}{P}} \frac{u}{T}\right) ; x_{0}=\left(\frac{E\left[u^{2}\right]}{T^{2}}+\frac{N_{0}}{2 P T}\right)^{-\frac{1}{2}},
$$

where $x_{0}$ is a normalization factor for $x$. The average error probability is given by

$$
P(E)=P\left(x<-x_{0}\right)=\int_{-\infty}^{-x_{0}} f_{x}(x) \mathrm{d} x
$$

where $f_{x}(x)$ is the pdf of the RV $x$. This can be expanded in the form

$$
f_{x}(x)=\frac{1}{\sqrt{2 \pi}} \exp \left(-\frac{x^{2}}{2}\right) \sum_{n=0}^{\infty} c_{n} H e_{n}(x)
$$

where $H e_{n}(x)$ is the $n$th Hermite polynomial

$$
H e_{n}(x)=(-1)^{n} \exp \left(-\frac{x^{2}}{2}\right) \frac{\mathrm{d}^{n}}{\mathrm{~d} x^{n}} \exp \left(-\frac{x^{2}}{2}\right) .
$$

The coefficients $c_{n}$ are obtained from the orthogonality relation of Hermite polynomials, and are given by $c_{n}=$ $E_{n} / n$ !, where $E_{n}=E\left[\operatorname{He}_{n}(x)\right]$. The coefficients $E_{n}$ are generalized moments, and can be written in terms of the central moments of $x$. Since the pdf of $x$ is an even function, and $H_{n}(x)$ is odd when $n$ is odd, $E_{n}$ is zero for $n$ odd. The generalized moments $E_{2 n}^{\prime}$ can be expressed in the form

$$
E_{2 n}=\sum_{n=0}^{n} h_{2 n, 2 k} E\left[x^{2 k}\right]
$$

where $h_{2 n, 2 k}$, the coefficients of the orthogonal polynomials $\mathrm{He}_{2 n}(x)$, can be computed using the recursion

$$
\begin{array}{ll}
h_{2 n, 2 k}=-\frac{n(2 n-1)}{n-k} h_{2 n-2,2 k}, & k=0, \ldots, n-1 \\
h_{2 n, 2 n}=1, & \text { for all } n
\end{array}
$$

The coefficients $E_{2 n}$ can be computed from [13]

$E\left[x^{2 k}\right]=x_{0}^{-2 k} \sum_{i=0}^{k}\left(\begin{array}{c}2 k \\ 2 i\end{array}\right) \frac{E\left[u^{2 i}\right]}{T^{2 i}} \frac{(2 k-2 i) !}{2^{k-i}(k-i) !}\left(\frac{N_{0}}{2 P T}\right)^{k-i}$

By substituting (8) into (7), and using the previous results, we obtain a series expansion for the average error probability:

$$
P(E)=Q\left(x_{0}\right)-\mathrm{e}^{-x_{0}^{2} / 2} \sum_{n=1}^{\infty} \frac{E_{2 n}}{(2 n) ! \sqrt{2 \pi}} H e_{2 n-1}\left(-x_{0}\right) .
$$

The first term is the error probability we would achieve in the presence of an additive Gaussian disturbance whose power spectral density is the sum of the multiple-access interference and of the Gaussian noise.

This technique provides satisfactory results when the number of users is large and the signal-to-Gaussian noise ratio is low [13]. In this situation, two to three terms in the summation are enough for a good approximation to error probability. The moments of the RV $u$ can be computed as described in [14].

\section{Gaussian assumption}

When the number of users is large enough, the pdf of the multiple interference is often assumed to approximately Gaussian, with a variance equal to the sum 
of the variances of the multiple-access interferers and of the Gaussian noise. The average error probability based on the Gaussian assumption can be expressed as

$$
P(E)=Q\left[\left(\frac{N_{0}}{2 E_{b}}+\frac{K-1}{3 N}\right)^{-\frac{1}{2}}\right] .
$$

\section{Choosing the code sequences}

A class of binary code sequences with good correlation properties is provided by the maximum-length sequences. Another choice is suggested by Pursley and Roefs [10]: among all the maximum-length sequences with period $N=2^{n}-1$, we retain only those whose correlation sidelobes have lower energies: these sequences are called AO/LSE (auto-optimal/least sidelobe energy). The main drawback with these sequences is the small number of them available for each value of $N$. Another possible choice is provided by Gold sequences [7].

\section{NUMERICAL RESULTS}

In this work we consider a pseudo-random sequence with length $N=255$ generated by an 8-stage shift register whose feedback connections are described by the polynomial 453 (octal notation). We assume that each user is assigned a shifted version of the sequence, so that a maximum of 255 users can be accommodated by the system.

Fig. 1 shows the average error probability of the system versus the number of users for several values of signal-to-Gaussian noise ratio $E_{b} / N_{0}$. The curves are obtained through the Gram-Charlier expansion method described before. The asterisks denote the approximation obtained under the Gaussian assumption, and refer to the error probability curve lying above them. It can be seen that the Gaussian assumption provides accurate enough results when the number of users is very large, and hence for high $P(E)$ values. The curves were obtained by retaining three terms in summation (10), and taking moments of the RV $u$ up to order six.

Fig. 2 reorganizes the results by showing the number of users that can access the system at the same time, as a function of the signal-to-Gaussian noise ratio for several values of error probability. We can see that, if an error probability less that $10^{-6}$ is sought, we need an $E_{b} / N_{0}$ of at least $20 \mathrm{~dB}$ for 25 simultaneous users. If each user transmits at a speed of $64000 \mathrm{bit} / \mathrm{s}$ and the processing gain is 255 ( $24 \mathrm{~dB}$ ), the bandwidth used is $64000 \times 255=16.3 \mathrm{MHz}$, that is, each user needs a bandwidth of about $650 \mathrm{KHz}$. Under these conditions CDMA multiple access offers no advantage with

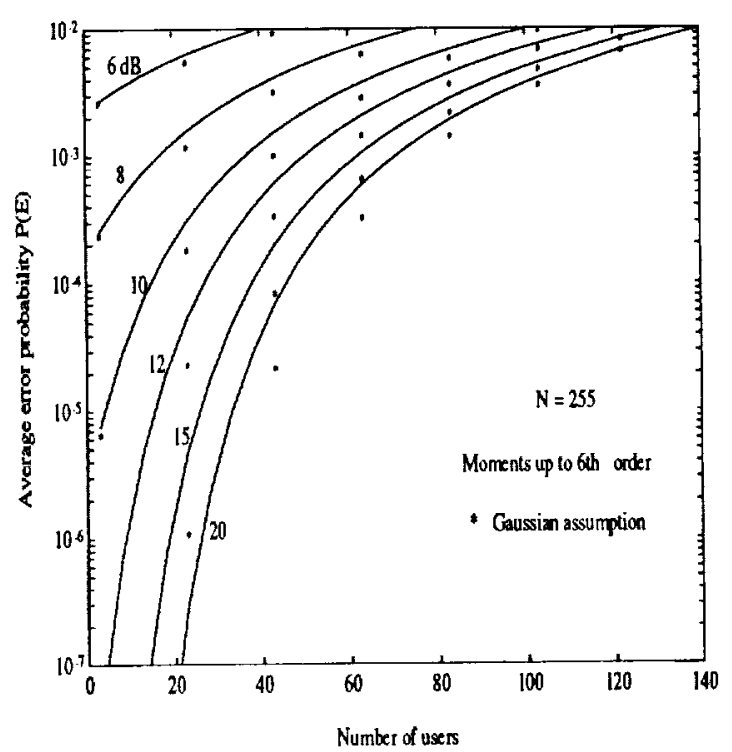

Figure 1: Average error probability $\mathrm{P}(\mathrm{E})$ versus the number of users for different values of the ratio $E_{b} / N_{0}$ for maximum-length sequences with $N=255$.

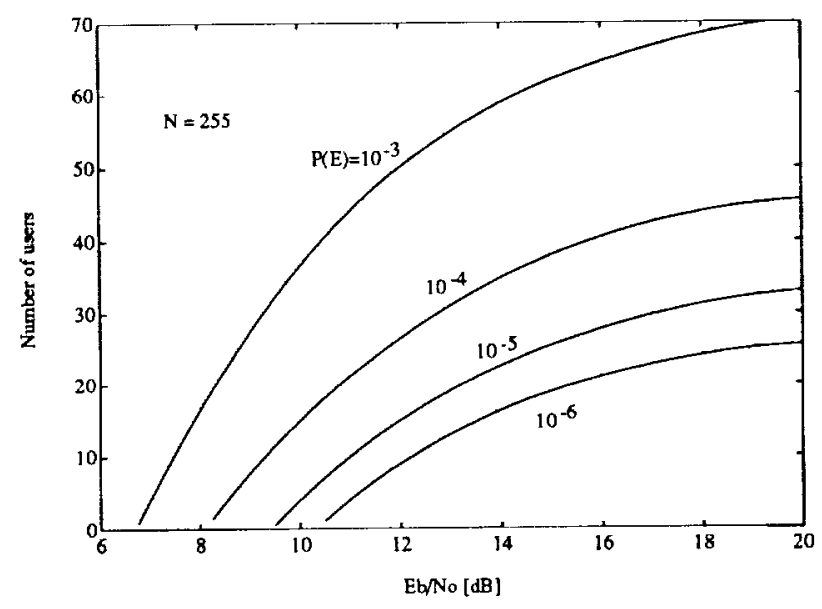

Figure 2: Number of simultaneous system users vs. $E_{b} / N_{0}$. The length of the code sequences is 255 .

respect to FDMA, which would need about $128 \mathrm{KHz}$ to transmit at $64 \mathrm{Kbit} / \mathrm{s}$. Thus, SSMA would need an efficient coding scheme (which provides a suitable coding gain) to become attractive.

To increase the number of users that can access the system while keeping the same error performance we may think of using longer sequences, which will increase processing gain. The average error probability $P(E)$ obtained with a sequence of length 511 (feedback shift register with nine stages and generating polyno- 
mial 1021) was computed. The corresponding values of system capacity vs. $E_{b} / N_{0}$ are shown in Fig. 3. We see that to achieve $K=25$ and $P(E)=10^{-6}$ as before, $13 \mathrm{~dB}$ are enough, with a savings of about $7 \mathrm{~dB}$. The cost is a twofold increase in bandwidth occupancy.

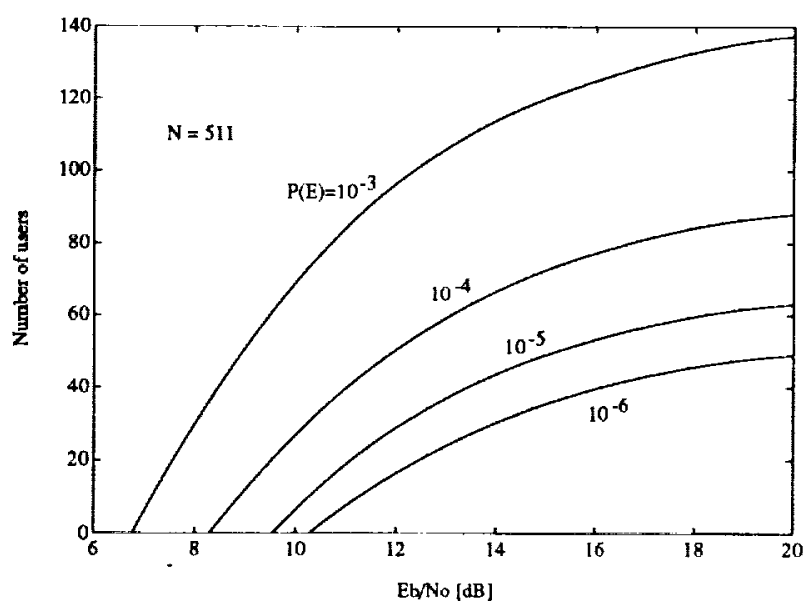

Figure 3: Number of users that can access the system with an assigned performance vs. $E_{b} / N_{0}$. The sequence length is 511 .

\section{Consideration of the satellite channel}

We now consider some of the major factors that increase the capacity of an SSMA system [6], viz., voice activity, spatial discrimination provided by crosspolarization frequency reuse, and multi-beam antennas.

Voice activity. With a $35 \%$ voice activity factor, the number of users in Fig. 2 and Fig. 3 must be multiplied by $1 / 0.35=2.86$. Thus, when $N=255$, for $P(E)=10^{-6}$ and $E_{b} / N_{0}=20 \mathrm{~dB}$ we have about 70 users, and the bandwidth is $650000 \times 0.35=227.5 \mathrm{Kz}$ per channel. With $N=511$, for the same values of $P(E)$ and $E_{b} / N_{0}$ we have about 140 users, and the bandwidth per channel is about the same.

Cross-polarization frequency reuse. As for frequency reuse by cross-polarization, a quantitative analysis can be performed by using the computational techniques described before. Assume that $K$ users access the system with each polarization. If $X_{\mathrm{p}}$ denotes the relative signal level with a given polarization, and received by a user transmitting with the opposite polarization, the total signal received by the $k$ th user is

$$
r_{k}(t)=\sqrt{2 P} \sum_{k=1}^{K} A_{k}(t)+X_{\mathrm{p}} \sqrt{2 P} \sum_{k=K+1}^{2 K} A_{k}(t)
$$

where

$$
A_{k}(t)=a_{k}\left(t-\tau_{k}\right) b_{k}\left(t-\tau_{k}\right) \cos \left(2 \pi f_{c} t+\phi_{k}\right) .
$$

The output of the $k$ th-user correlator is

$$
Z_{1}=\sqrt{\frac{P}{2}} T b_{1,0}+\sqrt{\frac{P}{2}} u+X_{\mathrm{p}} \sqrt{\frac{P}{2}} u^{\prime}+n
$$

where the first term in the right-hand side is useful signal, the second the interference from the users with the same polarization, and the third the interference from the cross-polarized users. As usual, $n$ is Gaussian noise. The RV $u$ was defined in (3), while $u^{\prime}$ is defined as follows:

$$
\begin{aligned}
u^{\prime} & =\sum_{k=K+1}^{2 K}\left[b_{k,-1} R_{k, 1}\left(\tau_{k}\right)+b_{k, 0} \hat{R}_{k, 1}\left(\tau_{k}\right)\right] \cos \phi_{k} \\
& =\sum_{k=K+1}^{2 K} w_{k} \cos \phi_{k}
\end{aligned}
$$

The two RVs $u$ and $u^{\prime}$ are independent, because $b_{k}$, $\tau_{k}$, and $\phi_{k}$ are independent for each user. If we define the new RV $v=u+X_{\mathrm{p}} u^{\prime}$, we obtain for error probability the same expression as (5), and hence to compute it we can use the same technique as before. To evaluate the moments of $v$ we follow [8], and obtain

$$
E\left[v^{2 n}\right]=\sum_{p=0}^{n}\left(\begin{array}{l}
2 n \\
2 p
\end{array}\right) E\left[u^{2 p}\right] X_{\mathrm{p}}^{2 n-2 p} E\left[u^{2 n-2 p}\right]
$$

where the moments of $u$ and $u^{\prime}$ are calculated as above.

The error probability obtained with a crosspolarized channel attenuation equal to $6 \mathrm{~dB}$, and sequence length 255 was computed. The corresponding values of capacity vs. $E_{b} / N_{0}$ are shown in Fig. 4 . We can see that the capacity increase is about $85 \%$, which could not be obtained by FDMA.

Fig. 5 shows capacity curves with sequence length 255 , cross-polarization diversity, and a $35 \%$ voice activity factor.

If error-control coding is used, these curves move toward left by an amount equal to the coding gain.

Spatial discrimination Channel capacity can be further increased if multi-beam antennas are used, which allow a degree of frequency reuse. The amount of reuse depends on the maximum interference that can be tolerated among beams transmitting in the same bandwidth. A factor of frequency reuse equal to 2 or 3 seems reasonable, which implies increasing the capacity by a factor 2 or 3 if the interference is negligible. 


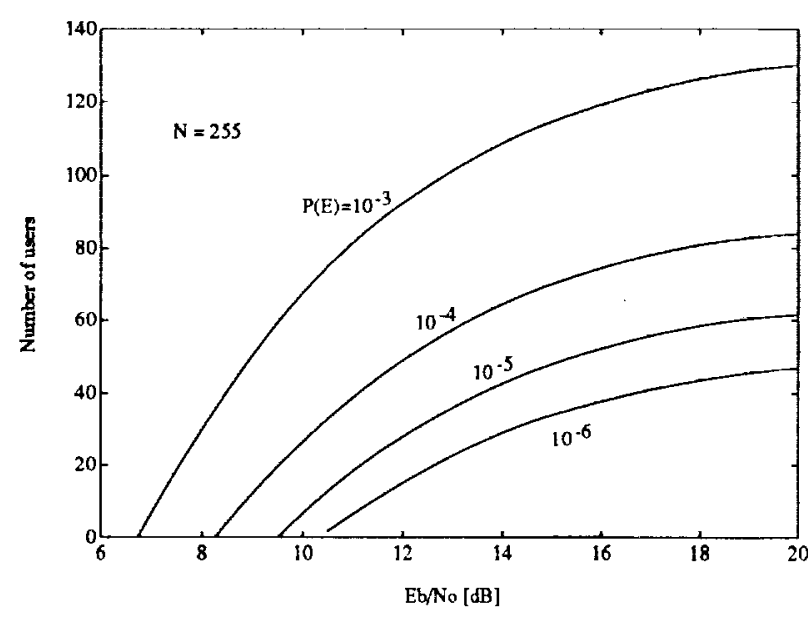

Figure 4: Number of users that can access the system with a given $P(E)$ for an attenuation of the crosspolarized channel equal to $6 \mathrm{~dB}$.

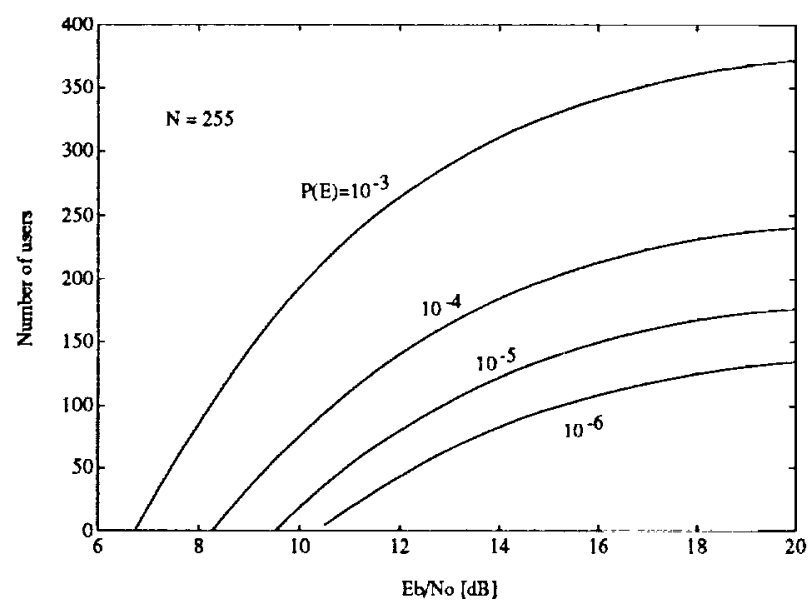

Figure 5: System capacity with single beam for several values of average error probability, an attenuation of the cross-polarized channel equal to $6 \mathrm{~dB}$, and $35 \%$ voice activity factor vs. $E_{b} / N_{0}$.

\section{SYSTEM CAPACITY}

Consider now the satellite mobile radio system described in the Introduction. Assume 1000 channels with 40 beams, i.e., 25 channels per beam. From Fig. 6, obtained from Fig. 5 by zooming in the relevant region of $E_{b} / N_{0}$ and $P(E)$ values, we can see that for 25 users to access the system with $P(E)=10^{-6}$ we need $E_{b} / N_{0} \simeq 11,3 \mathrm{~dB}$. With code sequences of length $N=511$, we need about $10.8 \mathrm{~dB}$ to achieve the same

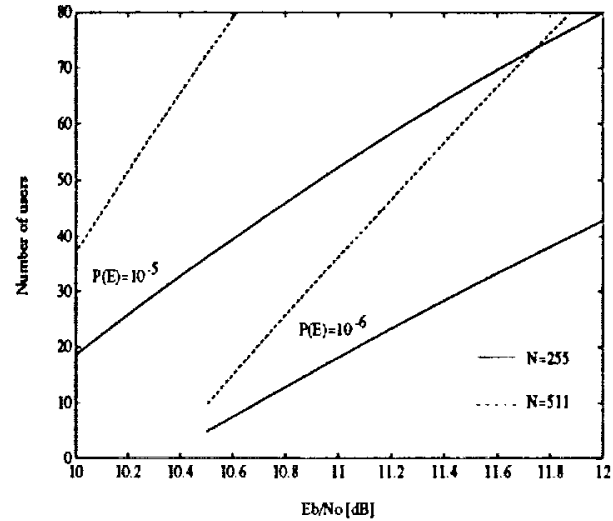

Figure 6: System capacity per beam for relevant values of $P(E)$ and $E_{b} / N_{0}$.

number of channels. By doubling the bandwidth, the achievable improvement is only $0.5 \mathrm{~dB}$.

\section{A note on synchronous SSMA}

To achieve a more efficient use of the bandwidth with spread-spectrum modulation, multiple-access noise due to asynchronous transmission should be eliminated. This may be accomplished by making the users' spreading modulation synchronous at the satellite, thus providing channels that are ideally orthogonal. In synchronous design, the users' signal would have to be synchronized to a small fraction of a chip time to achieve the desired orthogonality and minimization of interference. In this system, timing control algorithms, their accuracy, and their impact on system design are be important issues that will not be dealt with here. In the following, capacity evaluations are extended to synchronous SSMA.

With a fully synchronous system the delays $\tau_{k}$ in the system model can be disregarded, and we assume $\tau_{k}=0$ for $k=1,2, \ldots, k$. The signal received by the $k$ th user is

$$
r_{k}(t)=\sqrt{2 P} \sum_{k=1}^{K} a_{k}(t) b_{k}(t) \cos \left(2 \pi f_{c} t+\theta_{k}\right)+n_{k}(t)
$$

and the output from the correlator is

$$
Z_{1}=\sqrt{\frac{P}{2}} b_{1,0} T+\sqrt{\frac{P}{2}} w+n
$$

where the $\mathrm{RV} w$ is defined as

$$
w=\sum_{k=2}^{K} b_{k, 0} R_{k, 1}(0) \cos \theta_{k}
$$


with

$$
R_{k, 1}(0)=\int_{0}^{T} a_{k}(t) a_{1}(t) \mathrm{d} t
$$

Apart from a factor $1 / T, R_{k, 1}$ is the crosscorrelation between a pseudo-random sequence and its translated version,. It is well known that $R_{k, 1}(0)$ takes values $-T / N=-T_{c}$, with $N$ the sequence length. Thus, the RV $w$ can be given the form

$$
w=T \sum_{k=2}^{K} b_{k, 0} \cos \theta_{k}
$$

and is the sum of $K-1$ independent RVs.

To evaluate error probability, we compute the moments of $w$ and apply the Gram-Charlier expansion method as before. Fig. 7 compares the error probability of a system with 250 users and that of a channel with a single user and additive white Gaussian noise as the only disturbance. If an error probability $10^{-6}$ is desired, we need only to increase the signal-tonoise ratio by $0.2 \mathrm{~dB}$ with respect to the Gaussian channel. The spectral efficiency increases considerably, as we can allocate 250 users in the bandwidth $64000 \times 255=16,3 \mathrm{MHz}$.

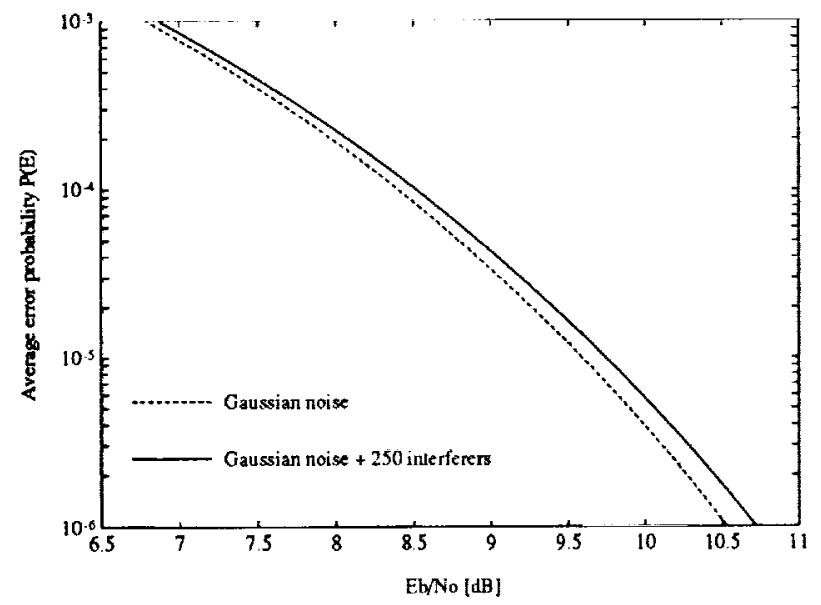

Figure 7: Average error probability vs. $E_{b} / N_{0}$ with synchronous transmission and sequence length 255 .

\section{References}

[1] S. Benedetto, E. Biglieri, and V. Castellani, Digital Transmission Theory. Englewood Cliffs, New Jersey: Prentice-Hall Inc., 1987.

[2] S. Benedetto, E. Biglieri, A. Luvison, and V. Zingarelli, "Moment-based evaluation of digital transmission system performance," IEE Proceedings-I, Vol. 139, No. 3, pp. 258-266, June 1992.
[3] C. Caini, G. Falciasecca, F. Valdoni, and F. Vatalaro, "A cellular satellite system conceived to enhance capabilities of a terrestrial cellular system," Global Satellite Communications Symposium, Nanjing, China, 1991.

[4] G. Falciasecca, A. Paraboni, M. Ruggieri, F. Valdoni, and F. Vatalaro, "Feasibility of an EHF (40/50 GHz) mobile satellite system using highly inclined orbits," Proc. IMSC'90, Ottawa, pp. 131-135, June 1990.

[5] R. S. Freeman, "On Gram-Charlier approximation", IEEE Trans. on Commun., Vol. COM-49, pp. 122125, February 1981.

[6] K. S. Gilhousen, I. M. Jacobs, R. Padovani, and L. A. Weaver, $\mathrm{J}_{\mathrm{r}}$, "Increased capacity using CDMA for mobile satellite communications", IEEE Journal on Selected Areas in Communications, Vol. 8, N. 4, pp. 503-514, May 1990.

[7] R. Gold, "Optimal binary sequences for spreadspectrum multiplexing", IEEE Trans, on Information Theory, Vol. IT-13, pp. 619-621, Ottobre 1967.

[8] V. K. Prabhu, "Some considerations of error bounds in digital systems", Bell System Technical Journal, Vol. 50, pp. 3127-3151, 1971.

[9] M. B. Pursley, D. V. Sarwate, and W. Stark, "Error probability for direct sequence spread spectrum multiple-access communications - Part I: Upper and lower bounds", IEEE Trans. on Commun., Vol. COM30, pp. 975-984, May 1982.

[10] M. B. Pursley and H. F. A. Roefs, "Numerical evaluation of correlation parameters of optimal phases of binary shift-register sequences", IEEE Trans. on Commun., Vol. COM-27, pp. 1597-1604, October 1979.

[11] M. B. Pursley, "Performance evaluation for phase coded SSMA communications - Part I: System analysis", IEEE Trans. on Commun., Vol. COM-25, N. 8, pp. 795-799, August. 1977.

[12] F. Valdoni, M. Ruggieri, F. Vatalaro, and A. Paraboni, "A new millimetre wave satellite system for land mobile communications," European Transactions on Telecommunications, Vol. I, N. 5, pp. 533544, September-October 1990.

[13] K. T. Wu, "Bandwidth utilisation of direct sequence spread-spectrum system", Proceedings of International Symposium on Communications, Tainan, Taiwan, R.O.C., December 9-13, 1991.

[14] K. T. Wu, "Average error probability for DS-SSMA communication systems", in Proc. of the 18th Annual Allerton Conference Commun., Control, Comput., pp. 359-368, October 1981. 